\title{
Editorial
}

\section{Ultrasound-guided regional anaesthesia: a new era in the field of anaesthesia}

The use of ultrasound for regional anesthesia is relatively new, however interest in this application is growing rapidly. Ultrasound guided nerve blocks were first described as early as 1978, but it was not until the advent of advanced ultrasound technology in the 1990's that interest in this field grew. Published reports of ultrasound guided regional anesthesia have largely focused on brachial plexus blockade in the interscalene, supraclavicular, infraclavicular and axillary regions. Recent studies examining the efficacy of ultrasound guidance for femoral, sciatic, psoas compartment, coeliac plexus and stellate ganglion blocks are promising, while ultrasound visualization of the epidural space can facilitate neuraxial blockade in children, adults and parturients.

Regional anesthesia performed with ultrasound guidance has become standard practice and has been endorsed by the National Institute of Clinical Excellence (NICE) in the UK. It can provide direct, real-time images of peripheral nerves and identify tissue planes that help to place the local anesthetic accurately. Ultrasound machines have improved substantially with the availability of high-resolution portable machines. Some even consider that ultrasound may soon become a part of the standard anesthesia machine. ${ }^{1}$ Do we know whether the use of ultrasound is safe and better than the other techniques for nerve block, and is there need for an evidence-base to show this, as some have voiced previously? ${ }^{2}$ Meanwhile, others suggest that the introduction of ultrasound is a step forward that is not required. ${ }^{3}$ To address this, there is now an attempt to establish a registry in one department in the USA (Hospital for Special Surgery, New York), which will document the efficacy and outcomes of ultrasound-guided regional anesthesia in order to build a database of evidence. ${ }^{4}$ They enrolled 1169 patients undergoing ambulatory shoulder arthroscopy and recorded their pre and post-operative observations, including any postoperative neurological signs. The success rate of the interscalene and supraclavicular blocks was approximately $99.8 \%$, and the rate of complications, including that of neurological symptoms, was low. What is required is more outcome studies, such as the one by Liu et $\mathrm{al}^{4}$ and for various types of blocks, to build up an evidence-base of the safety of ultrasound use in regional anesthesia.

Conventional peripheral nerve block techniques that are performed without visual guidance are highly dependent on surface anatomical landmarks for localization of the target nerve. It is therefore not surprising that regional anesthetic techniques are associated with a reported failure rate of up to $20 \%$ presumably because of incorrect needle and/ or local anesthetic placement. Multiple trial-anderror attempts to locate the target nerve can lead to operator frustration, unwarranted patient pain, and time delay in the operating room, especially in patients with difficult anatomical landmarks. Imaging technology such as MRI and CT scan can successfully localize neural structures. However, ultrasound is the most practical imaging tool for regional anesthesia as it is portable, relatively easy to learn, moderately priced, and does not pose any radiation risk. Ultrasound provides real time imaging guidance during a nerve block procedure. The following are the advantages of ultrasound $\left.{ }^{5}: 1\right)$ Reveals the nerve location and the surrounding vascular, muscular, bony, and visceral structures.2) Provides real-time imaging guidance during needle advancement allowing for purposeful needle movement and proper adjustments in direction and depth.3) Images the local anesthetic spread pattern during injection.4) Improves the quality of sensory block, the onset time, and the success rate compared to nerve stimulator techniques (as shown in some clinical studies).4)Reduces the number of needle attempts for nerve localization which may prove to reduce the risk of nerve injury.5)Differentiates extravascular injection from unintentional intravascular injection.6)Differentiates extraneural injection from unintentional intraneural injection. 
Undoubtedly, ultrasound has illuminated our knowledge of regional anesthesia and increased our understanding of block success and neurologic complications. Additional studies are required to identify its role in teaching and certification. However, even with ultrasound guidance, there will be vascular puncture and other needle-related trauma, intravascular and intraneuronal injections, and failed blocks. Ultrasound-guided regional anesthesia is not a metaphysical experience, it is physics expertly applied to the art of neural blockade. As the physics and our expertise improve, so may our outcomes.

\section{Md. Ahsanul Habib}

Consultant Cardiac Anaesthesia, Square Hospital, Dhaka

\section{References:}

1. Gray AT. Ultrasound-guided regional anesthesia. Anesthesiology 2006;104:368-373

2. Denny NM, Harrop-Griffiths W. Location, location, location! Ultrasound imaging in regional anaesthesia. Br J Anaesth 2005;94:1-3

3. Hopkins PM. Ultrasound guidance as a gold standard in regional anaesthesia. Br J Anaesth 2007;98:299-301

4. Liu SS, Gordon MA, Shaw PM, Wilfred S, Shetty T, et al. A prospective clinical registry of ultrasound-guided regional anesthesia for ambulatory shoulder surgery. Anesthesia and Analgesia . 2010;111:617-623

5. Marhofer P, Chan VWS. Ultrasound-guided regional anesthesia:Current concepts and future trends.Anesthesia and Analgesia 2007;104:1265-9 